\title{
Article \\ A Hardy-Hilbert-Type Inequality Involving Parameters Composed of a Pair of Weight Coefficients with Their Sums
}

\author{
Bicheng Yang ${ }^{1}$, Shanhe $\mathrm{Wu}^{2, *(1)}$ and Xingshou Huang ${ }^{3}$ \\ 1 Institute of Applied Mathematics, Longyan University, Longyan 364012, China; bcyang@gdei.edu.cn \\ 2 Department of Mathematics, Longyan University, Longyan 364012, China \\ 3 School of Mathematics and Statistics, Hechi University, Yizhou 456300, China; hxs509@hcnu.edu.cn \\ * Correspondence: shanhewu@lyun.edu.cn or shanhewu@163.com
}

check for

updates

Citation: Yang, B.; Wu, S.; Huang, X. A Hardy-Hilbert-Type Inequality Involving Parameters Composed of a Pair of Weight Coefficients with Their Sums. Mathematics 2021, 9, 2950. https://doi.org/10.3390/ math9222950

Academic Editor: Gabriel Eduard Vilcu

Received: 3 November 2021 Accepted: 15 November 2021 Published: 18 November 2021

Publisher's Note: MDPI stays neutral with regard to jurisdictional claims in published maps and institutional affiliations.

Copyright: (c) 2021 by the authors. Licensee MDPI, Basel, Switzerland. This article is an open access article distributed under the terms and conditions of the Creative Commons Attribution (CC BY) license (https:/ / creativecommons.org/licenses/by/ $4.0 /)$.
Abstract: In this paper, we establish a new Hardy-Hilbert-type inequality involving parameters composed of a pair of weight coefficients with their sum. Our result is a unified generalization of some Hardy-Hilbert-type inequalities presented in earlier papers. Based on the obtained inequality, the equivalent conditions of the best possible constant factor related to several parameters are discussed, and the equivalent forms and the operator expressions are also considered. As applications, we illustrate how the inequality obtained can generate some new Hardy-Hilbert-type inequalities.

Keywords: weight coefficients; Hardy-Hilbert-type inequality; Euler-Maclaurin summation formula; equivalent conditions; operator expressions

\section{Introduction}

The classical Hardy-Hilbert's inequality asserted the following:

$$
\sum_{m=1}^{\infty} \sum_{n=1}^{\infty} \frac{a_{m} b_{n}}{m+n}<\frac{\pi}{\sin (\pi / p)}\left(\sum_{m=1}^{\infty} a_{m}^{p}\right)^{\frac{1}{p}}\left(\sum_{n=1}^{\infty} b_{n}^{q}\right)^{\frac{1}{q}}
$$

where $p>1, \frac{1}{p}+\frac{1}{q}=1, a_{m}, b_{n} \geq 0,0<\sum_{m=1}^{\infty} a_{m}^{p}<\infty$ and $0<\sum_{n=1}^{\infty} b_{n}^{q}<\infty$, and $\frac{\pi}{\sin (\pi / p)}$ is the best possible constant factor (cf. [1], Theorem 315).

A sharpened version of inequality (1) was included in [1] by Theorem 323, as follows.

$$
\sum_{m=1}^{\infty} \sum_{n=1}^{\infty} \frac{a_{m} b_{n}}{m+n-1}<\frac{\pi}{\sin (\pi / p)}\left(\sum_{m=1}^{\infty} a_{m}^{p}\right)^{\frac{1}{p}}\left(\sum_{n=1}^{\infty} b_{n}^{q}\right)^{\frac{1}{q}}
$$

The integral version of Hilbert's inequality reads as follows (cf. [1], Theorem 316).

$$
\int_{0}^{\infty} \int_{0}^{\infty} \frac{f(x) g(y)}{x+y} d x d y<\pi\left(\int_{0}^{\infty} f^{2}(x) d x \int_{0}^{\infty} g^{2}(y) d y\right)^{\frac{1}{2}}
$$

In 1998, by introducing an independent parameter $\lambda>0$, Yang [2,3] provided an extension of Hilbert's integral inequality with the best possible constant factor $B\left(\frac{\lambda}{2}, \frac{\lambda}{2}\right)$ as follows.

$$
\int_{0}^{\infty} \int_{0}^{\infty} \frac{f(x) g(y)}{(x+y)^{\lambda}} d x d y<B\left(\frac{\lambda}{2}, \frac{\lambda}{2}\right)\left[\int_{0}^{\infty} x^{1-\lambda} f^{2}(x) d x \int_{0}^{\infty} y^{1-\lambda} g^{2}(y) d y\right]^{\frac{1}{2}} .
$$

For $\lambda=1$, inequality (4) reduces to the Hilbert's integral inequality (3). 
Inspired by the idea of establishing inequality (4), in 2001, with the help of the Euler-Maclaurin summation formula, Yang [4] proved a generalization of Hardy-Hilbert's inequality (1), i.e., for $\lambda \in(0,4]$; the following inequality holds.

$$
\sum_{m=1}^{\infty} \sum_{n=1}^{\infty} \frac{a_{m} b_{n}}{(m+n)^{\lambda}}<B\left(\frac{\lambda}{2}, \frac{\lambda}{2}\right)\left(\sum_{m=1}^{\infty} m^{1-\lambda} a_{m}^{2} \sum_{n=1}^{\infty} n^{1-\lambda} b_{n}^{2}\right)^{\frac{1}{2}} .
$$

In 2002, Yang and Debnath [5] generalized inequality (5) to the following inequality:

$$
\sum_{m=1}^{\infty} \sum_{n=1}^{\infty} \frac{a_{m} b_{n}}{(A m+B n)^{\lambda}}<\left(\frac{1}{A B}\right)^{\frac{\lambda}{2}} B\left(\frac{\lambda}{2}, \frac{\lambda}{2}\right)\left(\sum_{m=1}^{\infty} m^{1-\lambda} a_{m}^{2} \sum_{n=1}^{\infty} n^{1-\lambda} b_{n}^{2}\right)^{\frac{1}{2}}
$$

where $\lambda \in(0,2], A, B>0$.

In 2006, Krnić and Pečarić [6] provided an extension of inequalities (1) and (4) by introducing parameters $\lambda_{i} \in(0,2](i=1,2), \lambda_{1}+\lambda_{2}=\lambda \in(0,4]$, as follows:

$$
\sum_{m=1}^{\infty} \sum_{n=1}^{\infty} \frac{a_{m} b_{n}}{(m+n)^{\lambda}}<B\left(\lambda_{1}, \lambda_{2}\right)\left[\sum_{m=1}^{\infty} m^{p\left(1-\lambda_{1}\right)-1} a_{m}^{p}\right]^{\frac{1}{p}}\left[\sum_{n=1}^{\infty} n^{q\left(1-\lambda_{2}\right)-1} b_{n}^{q}\right]^{\frac{1}{q}}
$$

where the constant factor $B\left(\lambda_{1}, \lambda_{2}\right)$ is the best possible, and the following:

$$
B(u, v)=\int_{0}^{\infty} \frac{t^{u-1}}{(1+t)^{u+v}} d t(u, v>0)
$$

is the classical Beta function. Obviously, for $\lambda=1, \lambda_{1}=\frac{1}{q}, \lambda_{2}=\frac{1}{p}$, inequality (7) reduces to the Hardy-Hilbert's inequality (1); for $p=q=2, \lambda_{1}=\lambda_{2}=\frac{\lambda}{2}$,(7) reduces to (6).

Recently, Huang, Wu and Yang [7] provided a half-discrete Hardy-Hilbert-type inequality, as follows:

$$
\begin{gathered}
\int_{0}^{\infty} \sum_{n=1}^{\infty} \frac{a_{n} f(x)}{(x+n-\eta)^{s}} d x \leq\left(k_{s}\left(s_{2}\right)\right)^{\frac{1}{p}}\left(k_{s}\left(s_{1}\right)\right)^{\frac{1}{q}} \\
\times\left\{\int_{0}^{\infty} x^{p\left[1-\left(\frac{s-s_{2}}{p}+\frac{s_{1}}{q}\right)\right]-1} f^{p}(x) d x\right\}^{\frac{1}{p}}\left\{\sum_{n=1}^{\infty}(n-\eta)^{q\left[1-\left(\frac{s-s_{1}}{q}+\frac{s_{2}}{p}\right)\right]-1} a_{n}^{q}\right\}^{\frac{1}{q}},
\end{gathered}
$$

where $p>1, \frac{1}{p}+\frac{1}{q}=1, \eta \in\left[0, \frac{1}{4}\right], s \in(0,4], s_{1} \in(0, s), s_{2} \in\left(0, \frac{3}{2}\right] \cap(0, s)$.

$$
k_{s}\left(s_{i}\right):=B\left(s_{i}, s-s_{i}\right)(i=1,2) \text {. }
$$

Inequalities (1)-(3) play an important role in analysis and applications (cf. [8-18]).

In 2016, by means of the techniques of real analysis, Hong et al. [19] considered some equivalent statements of the extensions of (1) with the best possible constant factor related to a few parameters.

Motivated by the above-mentioned inequalities (2), (7) and (8), in this paper, we establish a new inequality that contains parameters composed of a pair of weight coefficients $\eta_{1}$ and $\eta_{2}$ with their sum $\eta\left(\eta \in\left[0, \frac{1}{2}\right]\right)$. The obtained inequality is a unified generalization of inequalities (2) and (7), as well as a more accurate version of inequalities (2) and (7). The main technical approaches include the construction of weight coefficients and the use of Hermite-Hadamard's inequality and the Euler-Maclaurin summation formula for estimation. Based on the obtained inequality, the equivalent conditions of the best possible constant factor related to several parameters are discussed. As applications, we deal with some equivalent forms, the operator expressions and some special cases for the inequalities obtained in the main result.

The rest of the paper is organized as follows: In Section 2, we provide some necessary notations, formulas and lemmas. In Sections 3 and 4, we state our main results, while some 
new inequalities with their equivalent forms and the operator expressions are provided. In Section 5, we end the paper with some concluding remarks and future directions of this study.

\section{Preliminaries}

Let us first state the following specified conditions (C1) that we will use in what follows. We suppose that the following is the case.

(C1) $p>1, q>1, \frac{1}{p}+\frac{1}{q}=1, \mathrm{~N}=\{1,2, \ldots\}, \eta_{i} \in\left[0, \frac{1}{4}\right](i=1,2), \eta_{1}+\eta_{2}=\eta \in\left[0, \frac{1}{2}\right]$

$\lambda \in(0,3], \lambda_{i} \in\left(0, \frac{3}{2}\right] \cap(0, \lambda), k_{\lambda}\left(\lambda_{i}\right):=B\left(\lambda_{i}, \lambda-\lambda_{i}\right)(i=1,2)$. We also assume $a_{m}, b_{n} \geq 0$ such that the following is the case.

$$
0<\sum_{m=1}^{\infty}\left(m-\eta_{1}\right)^{p\left[1-\left(\frac{\lambda-\lambda_{2}}{p}+\frac{\lambda_{1}}{q}\right)\right]-1} a_{m}^{p}<\infty \text {, and } 0<\sum_{n=1}^{\infty}\left(n-\eta_{2}\right)^{q\left[1-\left(\frac{\lambda-\lambda_{1}}{q}+\frac{\lambda_{2}}{p}\right)\right]-1} b_{n}^{q}<\infty .
$$

Hereinafter, the Euler-Maclaurin summation formula will be very helpful for us to deal with the estimations of integrals, which is stated as follows (cf. $[4,5])$.

Let $f:[1, \infty) \mapsto \mathrm{R}$ be non-negative continuously differentiable function such that $\sum_{k=1}^{\infty} f(k)<\infty$ and $\int_{1}^{\infty} f(t) d t<\infty$; then, the following equality holds:

$$
\sum_{k=1}^{\infty} f(k)=\int_{1}^{\infty} f(t) d t+\frac{1}{2} f(1)+\int_{1}^{\infty} \rho(t) f^{\prime}(t) d t
$$

where $\rho(t)=t-[t]-\frac{1}{2}$. Furthermore, if $f \in C^{4}[1, \infty), f^{(r)}(\infty)=0, r=0,1,2,3,4$, $f^{(2 r)}(x)>0$ and $f^{(2 r-1)}(x)<0, r=1,2$, then the following inequality holds.

$$
-\frac{1}{12} f(1)<\int_{1}^{\infty} \rho(t) f(t) d t<-\frac{1}{12} f(1)+\frac{1}{720} f^{\prime \prime}(1)<0 .
$$

Lemma 1. Define the following weight coefficient.

$$
\omega\left(\lambda_{2}, m\right):=\left(m-\eta_{1}\right)^{\lambda-\lambda_{2}} \sum_{n=1}^{\infty} \frac{\left(n-\eta_{2}\right)^{\lambda_{2}-1}}{(m+n-\eta)^{\lambda}}(m \in \mathrm{N}) .
$$

For $\lambda_{1} \in(0, \lambda), \lambda_{2} \in\left(0, \frac{3}{2}\right] \cap(0, \lambda)$, we have the following inequalities:

$$
0<k_{\lambda}\left(\lambda_{2}\right)\left(1-O\left(\frac{1}{\left(m-\eta_{1}\right)^{\lambda_{2}}}\right)\right)<\omega\left(\lambda_{2}, m\right)<k_{\lambda}\left(\lambda_{2}\right)(m \in \mathrm{N}),
$$

where $O\left(\frac{1}{\left(m-\eta_{1}\right)^{\lambda_{2}}}\right)$ is indicated as follows.

$$
O\left(\frac{1}{\left(m-\eta_{1}\right)^{\lambda_{2}}}\right):=\frac{1}{k_{\lambda}\left(\lambda_{2}\right)} \int_{0}^{\frac{1-\eta_{2}}{m-\eta_{1}}} \frac{u^{\lambda_{2}-1}}{(1+u)^{\lambda}} d u(>0)(m \in \mathrm{N}) .
$$

Proof. For fixed $m \in \mathrm{N}$, we set the following real function.

$$
g(m, t):=\frac{\left(t-\eta_{2}\right)^{\lambda_{2}-1}}{(m-\eta+t)^{\lambda}}\left(t>\eta_{2}\right) .
$$

In the following, we prove inequality (10) by considering two cases. 
Case (i). If $\lambda_{2} \in(0,1) \cap(0, \lambda)$, then by $(-1)^{i} g^{(i)}(m, t)>0\left(t>\eta_{2} ; i=0,1,2\right)$, and an application of the Hermite-Hadamard's inequality, we find, by setting $u=\frac{t-\eta_{2}}{m-\eta_{1}}$, that the following is the case.

$$
\begin{aligned}
\omega\left(\lambda_{2}, m\right) & =\left(m-\eta_{1}\right)^{\lambda-\lambda_{2}} \sum_{n=1}^{\infty} g(m, n)<\left(m-\eta_{1}\right) \lambda-\lambda_{2} \int_{\frac{1}{2}}^{\infty} g(m, t) d t \\
& =\left(m-\eta_{1}\right) \lambda-\lambda_{2} \int_{\frac{1}{2}}^{\infty} \frac{t^{\lambda_{2}-1}}{\left(m-\eta_{1}+t-\eta_{2}\right)^{\lambda}} d t=\int_{\frac{\frac{1}{2}-\eta_{2}}{m-\eta_{1}}}^{\infty} \frac{u^{\lambda_{2}-1}}{(1+u)^{\lambda}} d u \\
& \leq \int_{0}^{\infty} \frac{u^{\lambda_{2}-1}}{(1+u)^{\lambda}} d u=B\left(\lambda_{2}, \lambda-\lambda_{2}\right)=k_{\lambda}\left(\lambda_{2}\right) .
\end{aligned}
$$

In view of the decreasingness property of the series and by letting $u=\frac{t-\eta_{2}}{m-\eta_{1}}$, we obtain the following:

$$
\begin{aligned}
\omega\left(\lambda_{2}, m\right) & =\left(m-\eta_{1}\right)^{\lambda-\lambda_{2}} \sum_{n=1}^{\infty} g(m, n)>\left(m-\eta_{1}\right)^{\lambda-\lambda_{2}} \int_{1}^{\infty} g(m, t) d t \\
& =\int_{\frac{1-\eta_{2}}{m-\eta_{1}}}^{\infty} \frac{u^{\lambda_{2}-1}}{(1+u)^{\lambda}} d u=B\left(\lambda_{2}, \lambda-\lambda_{2}\right)-\int_{0}^{\frac{1-\eta_{2}}{m-\eta_{1}}} \frac{u^{\lambda_{2}-1}}{(1+u)^{\lambda}} d u \\
& =k_{\lambda}\left(\lambda_{2}\right)\left(1-O\left(\frac{1}{\left(m-\eta_{1}\right)^{\lambda_{2}}}\right)\right)>0,
\end{aligned}
$$

where $O\left(\frac{1}{\left(m-\eta_{1}\right)^{\lambda_{2}}}\right)=\frac{1}{k_{\lambda}\left(\lambda_{2}\right)} \int_{0}^{\frac{1-\eta_{2}}{m-\eta_{1}}} \frac{u^{\lambda_{2}-1}}{(1+u)^{\lambda}} d u>0$, satisfying the following case.

$$
0<\int_{0}^{\frac{1-\eta_{2}}{m-\eta_{1}}} \frac{u^{\lambda_{2}-1}}{(1+u)^{\lambda}} d u<\int_{0}^{\frac{1-\eta_{2}}{m-\eta_{1}}} u^{\lambda_{2}-1} d u=\frac{1}{\lambda_{2}}\left(\frac{1-\eta_{2}}{m-\eta_{1}}\right)^{\lambda_{2}}(m \in \mathrm{N}) .
$$

Hence, we obtain inequality (10).

Case (ii). If $\lambda_{2} \in\left[1, \frac{3}{2}\right] \cap(0, \lambda)$, then by means of the Euler-Maclaurin summation formula, we have the following:

$$
\begin{aligned}
& \sum_{n=1}^{\infty} g(m, n)=\int_{1}^{\infty} g(m, t) d t+\frac{1}{2} g(m, 1)+\int_{1}^{\infty} \rho(t) g^{\prime}(m, t) d t \\
& \quad=\int_{\eta_{2}}^{\infty} g(m, t) d t-h(m),
\end{aligned}
$$

where $\rho(t):=t-[t]-\frac{1}{2}$ is a Bernoulli function of first order, and $h(m)$ is indicated as follows.

$$
h(m):=\int_{\eta_{2}}^{1} g(m, t) d t-\frac{1}{2} g(m, 1)-\int_{1}^{\infty} \rho(t) g^{\prime}(m, t) d t .
$$

Note that $-\frac{1}{2} g(m, 1)=\frac{-\left(1-\eta_{2}\right)^{\lambda_{2}-1}}{2(m-\eta+1)^{\lambda}}$. Integrating by parts, the following is the case.

$$
\begin{aligned}
\int_{\eta_{2}}^{1} g(m, t) d t & =\int_{\eta_{2}}^{1} \frac{\left(t-\eta_{2}\right)^{\lambda_{2}-1}}{(m-\eta+t)^{\lambda}} d t=\frac{1}{\lambda_{2}} \int_{\eta_{2}}^{1} \frac{d(t-\eta) 2^{\lambda_{2}}}{(m-\eta+t)^{\lambda}} \\
& =\left.\frac{1}{\lambda_{2}} \frac{\left(t-\eta_{2}\right)^{\lambda_{2}}}{(m-\eta+t)^{\lambda}}\right|_{\eta_{2}} ^{1}+\frac{\lambda}{\lambda_{2}} \int_{\eta_{2}}^{1} \frac{\left(t-\eta_{2}\right)^{\lambda_{2}}}{(m-\eta+t)^{\lambda+1}} d t \\
& =\frac{1}{\lambda_{2}} \frac{\left(1-\eta_{2}\right)^{\lambda_{2}}}{(m-\eta+1)^{\lambda}}+\frac{\lambda}{\lambda_{2}\left(\lambda_{2}+1\right)} \int_{\eta_{2}}^{1} \frac{d\left(t-\eta_{2}\right)^{\lambda_{2}+1}}{(m-\eta+t)^{\lambda+1}} \\
& >\frac{1}{\lambda_{2}} \frac{\left(1-\eta_{2}\right)^{\lambda_{2}}}{(m-\eta+1)^{\lambda}}+\frac{\lambda}{\lambda_{2}\left(\lambda_{2}+1\right)}\left[\frac{\left(t-\eta_{2}\right)^{\lambda_{2}+1}}{(m-\eta+t)^{\lambda+1}}\right]_{\eta_{2}}^{1} \\
& +\frac{\lambda(\lambda+1)}{\lambda_{2}\left(\lambda_{2}+1\right)(m-\eta+1)^{\lambda+2}} \int_{\eta_{2}}^{1}\left(t-\eta_{2}\right)^{\lambda_{2}+1} d t \\
& =\frac{1}{\lambda_{2}} \frac{\left(1-\eta_{2}\right)^{\lambda_{2}}}{(m-\eta+1)^{\lambda}}+\frac{\lambda}{\lambda_{2}\left(\lambda_{2}+1\right)} \frac{\left(1-\eta_{2}\right)^{\lambda_{2}+1}}{(m-\eta+1)^{\lambda+1}}+\frac{\lambda(\lambda+1)\left(1-\eta_{2}\right)^{\lambda_{2}+2}}{\lambda_{2}\left(\lambda_{2}+1\right)\left(\lambda_{2}+2\right)(m-\eta+1)^{\lambda+2}}
\end{aligned}
$$


Moreover, we have the following:

$$
\begin{aligned}
-g^{\prime}(m, t) & =-\frac{\left(\lambda_{2}-1\right)\left(t-\eta_{2}\right)^{\lambda_{2}-2}}{(m-\eta+t)^{\lambda}}+\frac{\lambda\left(t-\eta_{2}\right)^{\lambda_{2}-1}}{(m-\eta+t)^{\lambda+1}} \\
& =\frac{\left(1-\lambda_{2}\right)\left(t-\eta \eta_{2}\right)^{\lambda_{2}-2}}{(m-\eta+t)^{\lambda}}+\frac{\lambda\left(t-\eta_{2}\right)^{\lambda_{2}-2}}{(m-\eta+t)^{\lambda}}-\frac{\lambda\left(m-\eta_{1}\right)\left(t-\eta_{2}\right)^{\lambda_{2}-2}}{(m-\eta+t)^{\lambda+1}} \\
& =\frac{\left(\lambda+1-\lambda_{2}\right)\left(t-\eta_{2}\right)^{\lambda_{2}-2}}{(m-\eta+t)^{\lambda}}-\frac{\lambda\left(m-\eta_{1}\right)\left(t-\eta_{2}\right)^{\lambda_{2}-2}}{(m-\eta+t)^{\lambda+1}},
\end{aligned}
$$

and for $\lambda_{2} \in\left[1, \frac{3}{2}\right] \cap(0, \lambda)$, we obtain the following.

$$
(-1)^{i} \frac{d^{i}}{d t^{i}}\left[\frac{\left(t-\eta_{2}\right)^{\lambda_{2}-2}}{(m-\eta+t)^{\lambda}}\right]>0,(-1)^{i} \frac{d^{i}}{d t^{i}}\left[\frac{\left(t-\eta_{2}\right)^{\lambda_{2}-2}}{(m-\eta+t)^{\lambda+1}}\right]>0\left(t>\eta_{2} ; i=0,1,2,3,4\right) .
$$

Using the Euler-Maclaurin summation formulas for estimation provides the following:

$$
-\frac{1}{12} g(1)<\int_{1}^{\infty} \rho(t) g(t) d t<-\frac{1}{12} g(1)+\frac{1}{720} g^{\prime \prime}(1)<0,
$$

where $(-1)^{i} g^{(i)}(t)>0, g^{(i)}(\infty)=0(i=0,1,2,3,4)(t>0)$, and then by setting $a:=1-\eta_{2}$ $\left(\in\left[\frac{4}{5}, 1\right]\right)$, we obtain the following.

$$
\begin{gathered}
\left(\lambda+1-\lambda_{2}\right) \int_{1}^{\infty} \rho(t) \frac{\left(t-\eta_{2}\right)^{\lambda_{2}-2}}{(m-\eta+t)^{\lambda}} d t>-\frac{\left(\lambda+1-\lambda_{2}\right) a^{\lambda_{2}-2}}{12(m-\eta+1)^{\lambda}}, \\
-\left(m-\eta_{1}\right) \lambda \int_{1}^{\infty} \rho(t) \frac{\left(t-\eta_{2}\right)^{\lambda_{2}-2}}{(m-\eta+t)^{\lambda+1}} d t>\frac{\left(m-\eta_{1}\right) \lambda a^{\lambda_{2}-2}}{12(m-\eta+1)^{\lambda+1}}-\frac{\left(m-\eta_{1}\right) \lambda}{720}\left[\frac{\left(t-\eta_{2}\right)^{\lambda_{2}-2}}{(m-\eta+t)^{\lambda+1}}\right]^{\prime \prime} t=1 \\
>\frac{(m-\eta+1) \lambda-a \lambda}{12(m-\eta+1)^{\lambda+1}} a^{\lambda_{2}-2} \\
-\frac{(m-\eta+1) \lambda}{720}\left[\frac{(\lambda+1)(\lambda+2)}{(m-\eta+1)^{\lambda+3}} a^{\lambda_{2}-2}+\frac{2(\lambda+1)\left(2-\lambda_{2}\right)}{(m-\eta+1)^{\lambda+2}} a^{\lambda_{2}-3}+\frac{\left(2-\lambda_{2}\right)\left(3-\lambda_{2}\right)}{(m-\eta+1)^{\lambda+1}} a^{\lambda_{2}-4}\right] \\
=\frac{\lambda a^{\lambda_{2}-2}}{12(m-\eta+1)^{\lambda}}-\frac{\lambda a^{\lambda_{2}-1}}{12(m-\eta+1)^{\lambda+1}} \\
-\frac{\lambda}{720}\left[\frac{(\lambda+1)(\lambda+2)}{(m-\eta+1)^{\lambda+2}} a^{\lambda_{2}-2}+\frac{2(\lambda+1)\left(2-\lambda_{2}\right)}{(m-\eta+1)^{\lambda+1}} a^{\lambda_{2}-3}+\frac{\left(2-\lambda_{2}\right)\left(3-\lambda_{2}\right)}{(m-\eta+1)^{\lambda}} a^{\lambda_{2}-4}\right] .
\end{gathered}
$$

Furthermore, we obtain the following:

$$
h(m)>\frac{a \lambda_{2}-4}{(m-\eta+1) \lambda} h_{1}+\frac{\lambda a^{\lambda_{2}-3}}{(m-\eta+1)^{\lambda+1}} h_{2}+\frac{\lambda(\lambda+1) a^{\lambda_{2}-2}}{(m-\eta+1)^{\lambda+2}} h_{3},
$$

where $h_{i}(i=1,2,3)$ are indicated as follows.

$$
\begin{aligned}
& h_{1}:=\frac{a^{4}}{\lambda_{2}}-\frac{a^{3}}{2}-\frac{\left(1-\lambda_{2}\right) a^{2}}{12}-\frac{\lambda\left(2-\lambda_{2}\right)\left(3-\lambda_{2}\right)}{720}, \\
& h_{2}:=\frac{a^{4}}{\lambda_{2}\left(\lambda_{2}+1\right)}-\frac{a^{2}}{12}-\frac{(\lambda+1)\left(2-\lambda_{2}\right)}{360}, \text { and } \\
& h_{3}:=\frac{a^{4}}{\lambda_{2}\left(\lambda_{2}+1\right)\left(\lambda_{2}+2\right)}-\frac{\lambda+2}{720} .
\end{aligned}
$$

For $\lambda \in(0,3], \lambda_{2} \in\left[1, \frac{3}{2}\right] \cap(0, \lambda), a \in\left[\frac{3}{4}, 1\right]$, we find the following.

$$
h_{1}>\frac{a^{2}}{12 \lambda_{2}}\left[\lambda_{2}^{2}-(6 a+1) \lambda_{2}+12 a^{2}\right]-\frac{1}{120} \text {. }
$$


In view of $\frac{d}{d a}\left[\lambda_{2}^{2}-(6 a+1) \lambda_{2}+12 a^{2}\right]=6\left(4 a-\lambda_{2}\right)>0$, and the following:

$$
\begin{aligned}
& \frac{d}{d \lambda_{2}}\left[\lambda_{2}^{2}-(6 a+1) \lambda_{2}+12 a^{2}\right]=2 \lambda_{2}-(6 a+1) \\
& \leq 2 \cdot \frac{3}{2}-\left(6 \cdot \frac{3}{4}+1\right)=3-\frac{11}{2}<0
\end{aligned}
$$

we obtain the following case.

$$
\begin{aligned}
& h_{1} \geq \frac{(3 / 4)^{2}}{12(3 / 2)}\left[\left(\frac{3}{2}\right)^{2}-\left(6 \cdot \frac{3}{4}+1\right) \frac{3}{2}+12\left(\frac{3}{4}\right)^{2}\right]-\frac{1}{120}=\frac{3}{128}-\frac{1}{120}>0, \\
& h_{2}>a^{2}\left(\frac{4 a^{2}}{15}-\frac{1}{12}\right)-\frac{1}{90} \geq\left(\frac{3}{4}\right)^{2}\left[\frac{4}{15}\left(\frac{3}{4}\right)^{2}-\frac{1}{12}\right]-\frac{1}{90}=\frac{3}{80}-\frac{1}{90}>0, \\
& h_{3} \geq \frac{8 a^{4}}{105}-\frac{5}{720}=\frac{8(3 / 4)^{4}}{105}-\frac{1}{144}=\frac{27}{1120}-\frac{1}{144}>0,
\end{aligned}
$$

Therefore, we deduce that $h(m)>0$.

On the other hand, we also have the following case:

$$
\begin{aligned}
& \sum_{n=1}^{\infty} g(m, n)=\int_{1}^{\infty} g(m, t) d t+\frac{1}{2} g(m, 1)+\int_{1}^{\infty} \rho(t) g^{\prime}(m, t) d t \\
& =\int_{1}^{\infty} g(m, t) d t+H(m)
\end{aligned}
$$

where $H(m)$ is indicated as follows.

$$
H(m):=\frac{1}{2} g(m, 1)+\int_{1}^{\infty} \rho(t) g^{\prime}(m, t) d t
$$

We have obtained $\frac{1}{2} g(m, 1)=\frac{a^{\lambda_{2}-1}}{2(m-\eta+1)^{\lambda}}$ and the following.

$$
g^{\prime}(m, t)=-\frac{\left(\lambda+1-\lambda_{2}\right)\left(t-\eta_{2}\right)^{\lambda_{2}-2}}{(m-\eta+t)^{\lambda}}+\frac{\lambda\left(m-\eta_{1}\right)\left(t-\eta_{2}\right)^{\lambda_{2}-2}}{(m-\eta+t)^{\lambda+1}} .
$$

For $\lambda_{2} \in\left(0, \frac{3}{2}\right] \cap(0, \lambda), 0<\lambda \leq 3$, by utilizing the Euler-Maclaurin summation formula for estimation again, one obtains the following.

$$
\begin{aligned}
& -\left(\lambda+1-\lambda_{2}\right) \int_{1}^{\infty} \rho(t) \frac{\left(t-\eta_{2}\right)^{\lambda_{2}-2}}{(m-\eta+t)^{\lambda}} d t>0, \\
& \left(m-\eta_{1}\right) \lambda \int_{1}^{\infty} \rho(t) \frac{\left(t-\eta_{2}\right)^{\lambda_{2}-2}}{(m-\eta+t)^{\lambda+1}} d t>\frac{-\left(m-\eta_{1}\right) \lambda}{12(m-\eta+1)^{\lambda+1}} a^{\lambda_{2}-2}=\frac{-(m-\eta+1) \lambda+a \lambda}{12(m-\eta+1)^{\lambda+1}} a^{\lambda_{2}-2} \\
& =\frac{-\lambda}{12(m-\eta+1)^{\lambda}} a^{\lambda_{2}-2}+\frac{\lambda}{12(m-\eta+1)^{\lambda+1}} a^{\lambda_{2}-1}>\frac{-\lambda}{12(m-\eta+1)^{\lambda}} a^{\lambda_{2}-2} .
\end{aligned}
$$

The following is, thus, obtained.

$$
\begin{gathered}
H(m)>\frac{a^{\lambda_{2}-1}}{2(m-\eta+1)^{\lambda}}-\frac{\lambda a^{\lambda_{2}-2}}{12(m-\eta+1)^{\lambda}}=\left(\frac{a}{2}-\frac{\lambda}{12}\right) \frac{a^{\lambda_{2}-2}}{(m-\eta+1)^{\lambda}} \\
\geq\left(\frac{1}{2} \cdot \frac{3}{4}-\frac{3}{12}\right) \frac{a^{\lambda_{2}-2}}{(m-\eta+1)^{\lambda}}=\left(\frac{3}{8}-\frac{3}{12}\right) \frac{a^{\lambda_{2}-2}}{(m-\eta+1)^{\lambda}}>0,
\end{gathered}
$$

Hence, we obtain the following inequalities.

$$
\int_{1}^{\infty} g(m, t) d t<\sum_{n=1}^{\infty} g(m, n)<\int_{\eta_{2}}^{\infty} g(m, t) d t
$$

In view of the results obtained in Case (i), we obtain inequality (10). Lemma 1 is proved. 
Lemma 2. Under the assumptions described in (C1), we have the following more accurate HardyHilbert's inequality.

$$
\begin{aligned}
I & =\sum_{n=1}^{\infty} \sum_{m=1}^{\infty} \frac{a_{m} b_{n}}{(m+n-\eta)^{\lambda}}<B^{\frac{1}{p}}\left(\lambda_{2}, \lambda-\lambda_{2}\right) B^{\frac{1}{q}}\left(\lambda_{1}, \lambda-\lambda_{1}\right) \\
& \times\left\{\sum_{m=1}^{\infty}\left(m-\eta_{1}\right)^{p\left[1-\left(\frac{\lambda-\lambda_{2}}{p}+\frac{\lambda_{1}}{q}\right)\right]-1} a_{m}^{p}\right\} \frac{1}{p}\left\{\sum_{n=1}^{\infty}\left(n-\eta_{2}\right)^{q\left[1-\left(\frac{\lambda-\lambda_{1}}{q}+\frac{\lambda_{2}}{p}\right)\right]-1} b_{n}^{q}\right\} \frac{1}{q}
\end{aligned}
$$

Proof. By using the same method as the proof for inequalitie (10), for $\lambda_{1} \in\left(0, \frac{3}{2}\right] \cap(0, \lambda)$, $\lambda_{2} \in(0, \lambda), n \in \mathbf{N}$, we can derive the following inequalities.

$$
\begin{aligned}
& B\left(\lambda_{1}, \lambda-\lambda_{1}\right)\left[1-O\left(\frac{1}{\left(n-\eta_{2}\right)^{\lambda_{1}}}\right)\right] \\
& <\omega\left(\lambda_{1}, n\right):=\left(n-\eta_{2}\right)^{\lambda-\lambda_{1}} \sum_{m=1}^{\infty} \frac{\left(n-\eta_{2}\right)^{\lambda_{1}-1}}{(m+n-\eta)^{\lambda}}<B\left(\lambda_{1}, \lambda-\lambda_{1}\right) .
\end{aligned}
$$

By using the Hölder inequality (cf. [20]):

$$
\sum_{n=1}^{\infty} \sum_{m=1}^{\infty} K(m, n) A_{m} B_{n} \leq\left(\sum_{m=1}^{\infty} \sum_{n=1}^{\infty} K(m, n) A_{m}^{p}\right)^{\frac{1}{p}}\left(\sum_{n=1}^{\infty} \sum_{m=1}^{\infty} K(m, n) B_{n}^{q}\right)^{\frac{1}{q}}
$$

where $K(m, n), A_{m}, B_{n} \geq 0$, the following is the case.

$$
\begin{aligned}
I & =\sum_{n=1}^{\infty} \sum_{m=1}^{\infty} \frac{1}{(m+n-\eta)^{\lambda}}\left[\frac{\left(n-\eta_{2}\right)^{\left(\lambda_{2}-1\right) / p}}{\left(m-\eta_{1}\right)^{\left(\lambda_{1}-1\right) / q}} a_{m}\right]\left[\frac{\left(m-\eta_{1}\right)^{\left(\lambda_{1}-1\right) / q}}{\left(n-\eta_{2}\right)^{\left(\lambda_{2}-1\right) / p}} b_{n}\right] \\
& \leq\left\{\sum_{m=1}^{\infty} \sum_{n=1}^{\infty} \frac{1}{(m+n-\eta)^{\lambda}} \frac{\left(n-\eta_{2}\right)^{\lambda_{2}-1}}{\left(m-\eta_{1}\right)^{\left(\lambda_{1}-1\right)(p-1)}} a_{m}^{p}\right\}^{\frac{1}{p}}\left\{\sum_{n=1}^{\infty} \sum_{m=1}^{\infty} \frac{1}{(m+n-\eta)^{\lambda}} \frac{\left(m-\eta_{1}\right)^{\lambda_{1}-1}}{\left(n-\eta_{2}\right)^{\left(\lambda_{2}-1\right)(q-1)}} b_{n}^{q}\right\}^{\frac{1}{q}} \\
& =\left\{\sum_{m=1}^{\infty} \omega\left(\lambda_{2}, m\right)\left(m-\eta_{1}\right)^{p\left[1-\left(\frac{\lambda-\lambda_{2}}{p}+\frac{\lambda_{1}}{q}\right)\right]-1} a_{m}^{p}\right\}^{\frac{1}{p}}\left\{\sum_{n=1}^{\infty} \omega\left(\lambda_{1}, n\right)\left(n-\eta_{2}\right)^{q\left[1-\left(\frac{\lambda-\lambda_{1}}{q}+\frac{\lambda_{2}}{p}\right)\right]-1} b_{n}^{q}\right\}^{\frac{1}{q}} .
\end{aligned}
$$

Then, by using inequalities (10) and (12) with $p>1, q>1$, we deduce inequality (11). The proof of Lemma 2 is complete.

Remark 1. As consequences of inequality (11), we have the following results.

(i) From inequality (11), for $\lambda_{1}+\lambda_{2}=\lambda \in(0,3], 0<\lambda_{i} \leq \frac{3}{2}(i=1,2)$, we obtain the following:

$$
0<\sum_{m=1}^{\infty}\left(m-\eta_{1}\right)^{p\left(1-\lambda_{1}\right)-1} a_{m}^{p}<\infty, 0<\sum_{n=1}^{\infty}\left(n-\eta_{2}\right)^{q\left(1-\lambda_{2}\right)-1} b_{n}^{q}<\infty,
$$

and the following inequality.

$$
\sum_{n=1}^{\infty} \sum_{m=1}^{\infty} \frac{a_{m} b_{n}}{(m+n-\eta)^{\lambda}}<B\left(\lambda_{1}, \lambda_{2}\right)\left\{\sum_{m=1}^{\infty}\left(m-\eta_{1}\right)^{p\left(1-\lambda_{1}\right)-1} a_{m}^{p}\right\}^{\frac{1}{p}}\left\{\sum_{n=1}^{\infty}\left(n-\eta_{2}\right)^{q\left(1-\lambda_{2}\right)-1} b_{n}^{q}\right\}^{\frac{1}{q}}
$$

(ii) For $\lambda=1, \lambda_{1}=\frac{1}{q}, \lambda_{2}=\frac{1}{p}, \eta=1$, inequality (14) reduces to inequality (2);

Furthermore, for $\eta=\eta_{1}=\eta_{2}=0$, inequality (14) reduces to inequality (7). Hence, inequalities (11) and (14) are the generalizations of inequalities (2) and (7), respectively. 
(iii) It is easy to observe that, for $\lambda>0$ and $\frac{\lambda-\lambda_{2}}{p}+\frac{\lambda_{1}}{q}<\frac{1}{q}, \frac{\lambda-\lambda_{1}}{q}+\frac{\lambda_{2}}{p}<\frac{1}{p}$, one obtains the following:

$$
\begin{aligned}
& \sum_{n=1}^{\infty} \sum_{m=1}^{\infty} \frac{a_{m} b_{n}}{(m+n)^{\lambda}}<\sum_{n=1}^{\infty} \sum_{m=1}^{\infty} \frac{a_{m} b_{n}}{(m+n-\eta)^{\lambda}}<B^{\frac{1}{p}}\left(\lambda_{2}, \lambda-\lambda_{2}\right) B^{\frac{1}{q}}\left(\lambda_{1}, \lambda-\lambda_{1}\right) \\
& \times\left\{\sum_{m=1}^{\infty}\left(m-\eta_{1}\right)^{p\left[1-\left(\frac{\lambda-\lambda_{2}}{p}+\frac{\lambda_{1}}{q}\right)\right]-1} a_{m}^{p}\right\}^{\frac{1}{p}}\left\{\sum_{n=1}^{\infty}\left(n-\eta_{2}\right)^{q\left[1-\left(\frac{\lambda-\lambda_{1}}{q}+\frac{\lambda_{2}}{p}\right)\right]-1} b_{n}^{q}\right\}^{\frac{1}{q}} \\
< & B^{\frac{1}{p}}\left(\lambda_{2}, \lambda-\lambda_{2}\right) B^{\frac{1}{q}}\left(\lambda_{1}, \lambda-\lambda_{1}\right) \\
\times & \left\{\sum_{m=1}^{\infty} m^{p\left[1-\left(\frac{\lambda-\lambda_{2}}{p}+\frac{\lambda_{1}}{q}\right)\right]-1} a_{m}^{p}\right\}^{\frac{1}{p}}\left\{\sum_{n=1}^{\infty} n^{q\left[1-\left(\frac{\lambda-\lambda_{1}}{q}+\frac{\lambda_{2}}{p}\right)\right]-1} b_{n}^{q}\right\}^{\frac{1}{q}}
\end{aligned}
$$

which implies that inequalities (11) and (14) are more accurate versions by comparison with inequalities (2) and (7), respectively.

Lemma 3. If $\lambda_{1}+\lambda_{2}=\lambda \in(0,3], 0<\lambda_{i} \leq \frac{3}{2}(i=1,2)$, then the constant factor $B\left(\lambda_{1}, \lambda_{2}\right)$ in (14) is the best possible.

Proof. For any $0<\varepsilon<p \lambda_{1}$, we set the following case.

$$
\widetilde{a}_{m}:=\left(m-\eta_{1}\right)^{\lambda_{1}-\frac{\varepsilon}{p}-1}, \widetilde{b}_{n}:=\left(n-\eta_{2}\right)^{\lambda_{2}-\frac{\varepsilon}{q}-1}(m, n \in \mathrm{N})
$$

If there exists a constant $M \leq B\left(\lambda_{1}, \lambda_{2}\right)$ such that (14) is valid when we replace $B\left(\lambda_{1}, \lambda_{2}\right)$ by $M$, then by specifically performing a substitution of $a_{m}=\widetilde{a}_{m}$ and $b_{n}=\widetilde{b}_{n}$ in (14), we have the following.

$$
\widetilde{I}:=\sum_{n=1}^{\infty} \sum_{m=1}^{\infty} \frac{\widetilde{a}_{m} \widetilde{b}_{n}}{(m+n-\eta)^{\lambda}}<M\left[\sum_{m=1}^{\infty}\left(m-\eta_{1}\right)^{p\left(1-\lambda_{1}\right)-1} \widetilde{a}_{m}^{p}\right]^{\frac{1}{p}}\left[\sum_{n=1}^{\infty}\left(n-\eta_{2}\right)^{q\left(1-\lambda_{2}\right)-1} \widetilde{b}_{n}^{q}\right]^{\frac{1}{q}}
$$

By inequality (15) and the decreasingness property of series, we obtain the following case.

$$
\begin{aligned}
\widetilde{I} & <M\left\{\sum_{m=1}^{\infty}\left(m-\eta_{1}\right)^{p\left(1-\lambda_{1}\right)-1}\left(m-\eta_{1}\right)^{p \lambda_{1}-\varepsilon-p}\right\}^{\frac{1}{p}}\left[\sum_{n=1}^{\infty}\left(n-\eta_{2}\right)^{q\left(1-\lambda_{2}\right)-1}\left(n-\eta_{2}\right)^{q \lambda_{2}-\varepsilon-q}\right]^{\frac{1}{q}} \\
& =M\left[\left(1-\eta_{1}\right)^{-\varepsilon-1}+\sum_{m=2}^{\infty}\left(m-\eta_{1}\right)^{-\varepsilon-1}\right]^{\frac{1}{p}}\left[\left(1-\eta_{2}\right)^{-\varepsilon-1}+\sum_{n=2}^{\infty}\left(n-\eta_{2}\right)^{-\varepsilon-1}\right]^{\frac{1}{q}} \\
& <M\left[\left(1-\eta_{1}\right)^{-\varepsilon-1}+\int_{1}^{\infty}\left(x-\eta_{1}\right)^{-\varepsilon-1} d x\right]^{\frac{1}{p}}\left[\left(1-\eta_{2}\right)^{-\varepsilon-1}+\int_{1}^{\infty}\left(y-\eta_{2}\right)^{-\varepsilon-1} d y\right]^{\frac{1}{q}} \\
& =\frac{M}{\varepsilon}\left[\varepsilon\left(1-\eta_{1}\right)^{-\varepsilon-1}+\left(1-\eta_{1}\right)^{-\varepsilon}\right]^{\frac{1}{p}}\left[\varepsilon\left(1-\eta_{2}\right)^{-\varepsilon-1}+\left(1-\eta_{2}\right)^{-\varepsilon}\right]^{\frac{1}{q}} .
\end{aligned}
$$

By using inequality (12) and setting the following:

$$
\hat{\lambda}_{1}=\lambda_{1}-\frac{\varepsilon}{p} \in\left(0, \frac{3}{2}\right) \cap(0, \lambda)\left(0<\hat{\lambda}_{2}=\lambda_{2}+\frac{\varepsilon}{p}<\lambda\right),
$$

we find the following case.

$$
\begin{aligned}
\widetilde{I} & =\sum_{n=1}^{\infty}\left[\left(n-\eta_{2}\right)^{\left(\lambda_{2}+\frac{\varepsilon}{p}\right)} \sum_{m=1}^{\infty} \frac{1}{(m+n-\eta)^{\lambda}}\left(m-\eta_{1}\right)^{\left(\lambda_{1}-\frac{\varepsilon}{p}\right)-1}\right]\left(n-\eta_{2}\right)^{-\varepsilon-1} \\
& =\sum_{n=1}^{\infty} \omega\left(\hat{\lambda}_{1}, n\right)\left(n-\eta_{2}\right)^{-\varepsilon-1}>B\left(\hat{\lambda}_{1}, \hat{\lambda}_{2}\right) \sum_{n=1}^{\infty}\left[1-O\left(\frac{1}{\left(n-\eta_{2}\right)^{\lambda_{1}}}\right)\right]\left(n-\eta_{2}\right)^{-\varepsilon-1} \\
& =B\left(\hat{\lambda}_{1}, \hat{\lambda}_{2}\right)\left[\sum_{n=1}^{\infty}\left(n-\eta_{2}\right)^{-\varepsilon-1}-\sum_{n=1}^{\infty} O\left(\frac{1}{\left(n-\eta_{2}\right)^{\lambda_{1}+\varepsilon+1}}\right)\right] \\
& >B\left(\hat{\lambda}_{1}, \hat{\lambda}_{2}\right)\left[\int_{1}^{\infty}\left(y-\eta_{2}\right)^{-\varepsilon-1} d y-O(1)\right]
\end{aligned}
$$




$$
=\frac{1}{\varepsilon} B\left(\lambda_{1}-\frac{\varepsilon}{p}, \lambda_{2}+\frac{\varepsilon}{p}\right)\left[\left(1-\eta_{2}\right)^{-\varepsilon}-\varepsilon O(1)\right] .
$$

Then, we obtain the following.

$$
\begin{aligned}
& B\left(\lambda_{1}-\frac{\varepsilon}{p}, \lambda_{2}+\frac{\varepsilon}{p}\right)\left[\left(1-\eta_{2}\right)^{-\varepsilon}-\varepsilon O(1)\right] \\
& <\varepsilon \widetilde{I}<M\left[\varepsilon\left(1-\eta_{1}\right)^{-\varepsilon-1}+\left(1-\eta_{1}\right)^{-\varepsilon}\right]^{\frac{1}{p}}\left[\varepsilon\left(1-\eta_{2}\right)^{-\varepsilon-1}+\left(1-\eta_{2}\right)^{-\varepsilon}\right]^{\frac{1}{q}} .
\end{aligned}
$$

Now, letting $\varepsilon \rightarrow 0^{+}$at both sides of the above inequality, in view of the continuity of the Beta function, we find $B\left(\lambda_{1}, \lambda_{2}\right) \leq M$. Hence, $M=B\left(\lambda_{1}, \lambda_{2}\right)$ is the best possible constant factor in (14). This completes the proof for Lemma 3.

Remark 2. Setting $\widetilde{\lambda}_{1}:=\frac{\lambda-\lambda_{2}}{p}+\frac{\lambda_{1}}{q}, \widetilde{\lambda}_{2}:=\frac{\lambda-\lambda_{1}}{q}+\frac{\lambda_{2}}{p}$ in inequality (11), we find the following case:

$$
\widetilde{\lambda}_{1}+\widetilde{\lambda}_{2}=\frac{\lambda-\lambda_{2}}{p}+\frac{\lambda_{1}}{q}+\frac{\lambda-\lambda_{1}}{q}+\frac{\lambda_{2}}{p}=\frac{\lambda}{p}+\frac{\lambda}{q}=\lambda
$$

and the following is also the case.

$$
\widetilde{\lambda}_{1}=\frac{\lambda-\lambda_{2}}{p}+\frac{\lambda_{1}}{q}>0, \widetilde{\lambda}_{1}<\frac{\lambda}{p}+\frac{\lambda}{q}=\lambda, 0<\widetilde{\lambda}_{2}=\lambda-\widetilde{\lambda}_{1}<\lambda
$$

Hence, it follows that $B\left(\widetilde{\lambda}_{1}, \widetilde{\lambda}_{2}\right) \in \mathbf{R}_{+}=(0, \infty)$. Note that for the following case:

$$
\lambda-\lambda_{1}-\lambda_{2} \in\left[\left(\lambda-\lambda_{1}-\frac{3}{2}\right) p,\left(\frac{3}{2}-\lambda_{1}\right) p\right],
$$

we have $\widetilde{\lambda}_{1}, \widetilde{\lambda}_{2} \leq \frac{3}{2}$; thus, we can rewrite inequality (14) in the following form.

$$
\begin{aligned}
I= & \sum_{n=1}^{\infty} \sum_{m=1}^{\infty} \frac{a_{m} b_{n}}{(m+n-\eta)^{\lambda}}<B\left(\widetilde{\lambda}_{1}, \widetilde{\lambda}_{2}\right) \\
& \times\left\{\sum_{m=1}^{\infty}\left(m-\eta_{1}\right)^{p\left(1-\widetilde{\lambda}_{1}\right)-1} a_{m}^{p}\right\}^{\frac{1}{p}}\left\{\sum_{n=1}^{\infty}\left(n-\eta_{2}\right)^{q\left(1-\widetilde{\lambda}_{2}\right)-1} b_{n}^{q}\right\}^{\frac{1}{q}} .
\end{aligned}
$$

Lemma 4. If the constant factor $B^{\frac{1}{p}}\left(\lambda_{2}, \lambda-\lambda_{2}\right) B^{\frac{1}{q}}\left(\lambda_{1}, \lambda-\lambda_{1}\right)$ in (11) is the best possible, then for the following case:

$$
\lambda-\lambda_{1}-\lambda_{2} \in\left[\left(\lambda-\lambda_{1}-\frac{3}{2}\right) p,\left(\frac{3}{2}-\lambda_{1}\right) p\right],
$$

we have $\lambda_{1}+\lambda_{2}=\lambda$.

Proof. If the constant factor $B^{\frac{1}{p}}\left(\lambda_{2}, \lambda-\lambda_{2}\right) B^{\frac{1}{q}}\left(\lambda_{1}, \lambda-\lambda_{1}\right)$ in (11) is the best possible, then in view of the assumption in (11), we have the following.

$$
B^{\frac{1}{p}}\left(\lambda-\lambda_{2}, \lambda_{2}\right) B^{\frac{1}{q}}\left(\lambda_{1}, \lambda-\lambda_{1}\right) \leq B\left(\widetilde{\lambda}_{1}, \widetilde{\lambda}_{2}\right)
$$


By applying Hölder inequality, we have the following case:

$$
\begin{aligned}
& B\left(\widetilde{\lambda}_{1}, \widetilde{\lambda}_{2}\right)=B\left(\frac{\lambda-\lambda_{2}}{p}+\frac{\lambda_{1}}{q}, \frac{\lambda-\lambda_{1}}{q}+\frac{\lambda_{2}}{p}\right) \\
& =\int_{0}^{\infty} \frac{1}{(1+u)^{\lambda}} u^{\frac{\lambda-\lambda_{2}}{p}+\frac{\lambda_{1}}{q}-1} d u=\int_{0}^{\infty} \frac{1}{(1+u)^{\lambda}}\left(u^{\frac{\lambda-\lambda_{2}-1}{p}}\right)\left(u^{\frac{\lambda_{1}-1}{q}}\right) d u \\
& \leq\left[\int_{0}^{\infty} \frac{1}{(1+u)^{\lambda}} u^{\lambda-\lambda_{2}-1} d u\right]^{\frac{1}{p}}\left[\int_{0}^{\infty} \frac{1}{(1+u)^{\lambda}} u^{\lambda_{1}-1} d u\right]^{\frac{1}{q}} \\
& =B^{\frac{1}{p}}\left(\lambda-\lambda_{2}, \lambda_{2}\right) B^{\frac{1}{q}}\left(\lambda_{1}, \lambda-\lambda_{1}\right),
\end{aligned}
$$

and then we obtain $B\left(\widetilde{\lambda}_{1}, \widetilde{\lambda}_{2}\right)=B^{\frac{1}{p}}\left(\lambda-\lambda_{2}, \lambda_{2}\right) B^{\frac{1}{q}}\left(\lambda_{1}, \lambda-\lambda_{1}\right)$, namely, (17) retains the form of equality.

It is easily to observe that (17) keeps the form of equality if and only if there exist constants $A$ and $B$ such that they are not both zero and (cf. [20]) the following case holds.

$$
A u^{\lambda-\lambda_{2}-1}=B u^{\lambda_{1}-1} \text { a.e.in } \mathrm{R}_{+} .
$$

Assuming that $A \neq 0$, it follows that $u^{\lambda-\lambda_{2}-\lambda_{1}}=\frac{B}{A}$ a.e. in $\mathrm{R}_{+}$, which implies $\lambda-\lambda_{2}-$ $\lambda_{1}=0$, namely, $\lambda_{1}+\lambda_{2}=\lambda$. Lemma 4 is proved.

\section{Main Results}

Our main results are stated in the following theorems.

Theorem 1. Under the assumptions described in (C1), we have the following inequality that is equivalent to inequality (11).

$$
\begin{aligned}
& J:=\left\{\sum_{n=1}^{\infty}\left(n-\eta_{2}\right)^{p\left(\frac{\lambda-\lambda_{1}}{q}+\frac{\lambda_{2}}{p}\right)-1}\left[\sum_{m=1}^{\infty} \frac{1}{(m+n-\eta)^{\lambda}} a_{m}\right]^{p}\right\}^{\frac{1}{p}} \\
& <B^{\frac{1}{p}}\left(\lambda_{2}, \lambda-\lambda_{2}\right) B^{\frac{1}{q}}\left(\lambda_{1}, \lambda-\lambda_{1}\right)\left\{\sum_{m=1}^{\infty}\left(m-\eta_{1}\right)^{p\left[1-\left(\frac{\lambda-\lambda_{2}}{p}+\frac{\lambda_{1}}{q}\right)\right]-1} a_{m}^{p}\right\}^{\frac{1}{p}} .
\end{aligned}
$$

Furthermore, if the constant factor in (11) is the best possible; thus, so is the constant factor in (18).

Proof. Suppose that (18) is valid. By utilizing the Hölder inequality, we have the following case.

$$
\begin{aligned}
& I=\sum_{n=1}^{\infty}\left[\left(n-\eta_{2}\right)^{\frac{-1}{p}+\left(\frac{\lambda-\lambda_{1}}{q}+\frac{\lambda_{2}}{p}\right)} \sum_{m=1}^{\infty} \frac{1}{(m+n-\eta)^{\lambda}} a_{m}\right]\left[\left(n-\eta_{2}\right)^{\frac{1}{p}-\left(\frac{\lambda-\lambda_{1}}{q}+\frac{\lambda_{2}}{p}\right)} b_{n}\right] \\
& \leq J\left\{\sum_{n=1}^{\infty}\left(n-\eta_{2}\right)^{q\left[1-\left(\frac{\lambda-\lambda_{1}}{q}+\frac{\lambda_{2}}{p}\right)\right]-1} b_{n}^{q}\right\}^{\frac{1}{q}} .
\end{aligned}
$$

Then, by (18), we obtain (11). On the other hand, assuming that (11) is valid, we set the following.

$$
b_{n}:=\left(n-\eta_{2}\right)^{p\left(\frac{\lambda-\lambda_{1}}{q}+\frac{\lambda_{2}}{p}\right)-1}\left[\sum_{m=1}^{\infty} \frac{1}{(m+n-\eta)^{\lambda}} a_{m}^{p-1}, n \in \mathbf{N} .\right.
$$


If $J=0$, then (18) is naturally valid; if $J=\infty$, then it is impossible to make (18) valid, namely, $J<\infty$. Suppose that $0<J<\infty$. By (11), we have the following case:

$$
\begin{aligned}
& \sum_{n=1}^{\infty}\left(n-\eta_{2}\right)^{q\left[1-\left(\frac{\lambda-\lambda_{1}}{q}+\frac{\lambda_{2}}{p}\right)\right]-1} b_{n}^{q}=J^{p}=I<B^{\frac{1}{p}}\left(\lambda_{2}, \lambda-\lambda_{2}\right) B^{\frac{1}{q}}\left(\lambda_{1}, \lambda-\lambda_{1}\right) \\
& \times\left\{\sum_{m=1}^{\infty}\left(m-\eta_{1}\right)^{p\left[1-\left(\frac{\lambda-\lambda_{2}}{p}+\frac{\lambda_{1}}{q}\right)\right]-1} a_{m}^{p}\right\}^{\frac{1}{p}}\left\{\sum_{n=1}^{\infty}\left(n-\eta_{2}\right)^{q\left[1-\left(\frac{\lambda-\lambda_{1}}{q}+\frac{\lambda_{2}}{p}\right)\right]-1} b_{n}^{q}\right\}^{\frac{1}{q}}, \\
& J=\left\{\sum_{n=1}^{\infty}\left(n-\eta_{2}\right)^{q\left[1-\left(\frac{\lambda-\lambda_{1}}{q}+\frac{\lambda_{2}}{p}\right)\right]-1} b_{n}^{q}\right\}^{\frac{1}{p}} \\
& <B^{\frac{1}{p}}\left(\lambda_{2}, \lambda-\lambda_{2}\right) B^{\frac{1}{q}}\left(\lambda_{1}, \lambda-\lambda_{1}\right)\left\{\sum_{m=1}^{\infty}\left(m-\eta_{1}\right)^{p\left[1-\left(\frac{\lambda-\lambda_{2}}{p}+\frac{\lambda_{1}}{q}\right)\right]-1} a_{m}^{p}\right\}^{\frac{1}{p}}
\end{aligned}
$$

namely, inequality (18) holds true, which is equivalent to inequality (11).

If the constant factor in (11) is the best possible, then so is the constant factor in (18). Otherwise, by (19), we would reach a contradiction that the constant factor in (11) is not the best possible. This completes the proof for Theorem 1 .

Theorem 2. The following statements (i), (ii), (iii) and (iv) are equivalent:

(i) Both $B^{\frac{1}{p}}\left(\lambda_{2}, \lambda-\lambda_{2}\right) B^{\frac{1}{q}}\left(\lambda_{1}, \lambda-\lambda_{1}\right)$ and $B\left(\frac{\lambda-\lambda_{2}}{p}+\frac{\lambda_{1}}{q}, \frac{\lambda-\lambda_{1}}{q}+\frac{\lambda_{2}}{p}\right)$ are independent of $p, q$;

(ii) $B^{\frac{1}{p}}\left(\lambda_{2}, \lambda-\lambda_{2}\right) B^{\frac{1}{q}}\left(\lambda_{1}, \lambda-\lambda_{1}\right)$ is expressible as a convergent single integral:

$$
B\left(\frac{\lambda-\lambda_{2}}{p}+\frac{\lambda_{1}}{q}, \frac{\lambda-\lambda_{1}}{q}+\frac{\lambda_{2}}{p}\right)=B\left(\widetilde{\lambda}_{1}, \widetilde{\lambda}_{2}\right)=\int_{0}^{\infty} \frac{u^{\widetilde{\lambda}_{1}-1}}{(1+u)^{\lambda}} d u\left(\in \mathrm{R}_{+}\right) ;
$$

(iii) $B^{\frac{1}{p}}\left(\lambda_{2}, \lambda-\lambda_{2}\right) B^{\frac{1}{q}}\left(\lambda_{1}, \lambda-\lambda_{1}\right)$ in (11) is the best possible constant factor;

(iv) If $\lambda-\lambda_{1}-\lambda_{2} \in\left[\left(\lambda-\lambda_{1}-\frac{3}{2}\right) p,\left(\frac{3}{2}-\lambda_{1}\right) p\right]$, then $\lambda_{1}+\lambda_{2}=\lambda$.

If statement (iv) is valid, namely, $\lambda=\lambda_{1}+\lambda_{2}$, then we have (14) and the following equivalent inequality with the best possible constant factor $B\left(\lambda_{1}, \lambda_{2}\right)$.

$$
\left\{\sum_{n=1}^{\infty}\left(n-\eta_{2}\right)^{p \lambda_{2}-1}\left[\sum_{m=1}^{\infty} \frac{1}{(m+n-\eta)^{\lambda}} a_{m}\right]^{p}\right\}^{\frac{1}{p}}<B\left(\lambda_{1}, \lambda_{2}\right)\left[\sum_{m=1}^{\infty}\left(m-\eta_{1}\right)^{p\left(1-\lambda_{1}\right)-1} a_{m}^{p}\right]^{\frac{1}{p}} .
$$

Proof. (i) $\Rightarrow$ (ii). By (i), in view of the continuity of the Beta function, we have the following case:

$$
\begin{gathered}
B^{\frac{1}{p}}\left(\lambda_{2}, \lambda-\lambda_{2}\right) B^{\frac{1}{q}}\left(\lambda_{1}, \lambda-\lambda_{1}\right) \\
=\lim _{p \rightarrow 1^{+} q \rightarrow \infty} \lim ^{\frac{1}{p}}\left(\lambda_{2}, \lambda-\lambda_{2}\right) B^{\frac{1}{q}}\left(\lambda_{1}, \lambda-\lambda_{1}\right)=B\left(\lambda_{2}, \lambda-\lambda_{2}\right), \\
B\left(\frac{\lambda-\lambda_{2}}{p}+\frac{\lambda_{1}}{q}, \frac{\lambda-\lambda_{1}}{q}+\frac{\lambda_{2}}{p}\right) \\
=\lim _{p \rightarrow 1^{+}} \lim _{q \rightarrow \infty}\left(\frac{\lambda-\lambda_{2}}{p}+\frac{\lambda_{1}}{q}, \frac{\lambda-\lambda_{1}}{q}+\frac{\lambda_{2}}{p}\right)=B\left(\lambda-\lambda_{2}, \lambda_{2}\right)=B\left(\lambda_{2}, \lambda-\lambda_{2}\right),
\end{gathered}
$$

thus, $B^{\frac{1}{p}}\left(\lambda_{2}, \lambda-\lambda_{2}\right) B^{\frac{1}{q}}\left(\lambda_{1}, \lambda-\lambda_{1}\right)$ can be expressible as a convergent single integral.

$$
B\left(\frac{\lambda-\lambda_{2}}{p}+\frac{\lambda_{1}}{q}, \frac{\lambda-\lambda_{1}}{q}+\frac{\lambda_{2}}{p}\right)=\int_{0}^{\infty} \frac{u^{\widetilde{\lambda}_{1}-1}}{(1+u)^{\lambda}} d u\left(\in \mathrm{R}_{+}\right) .
$$


(ii) $\Rightarrow$ (iv). If $B^{\frac{1}{p}}\left(\lambda_{2}, \lambda-\lambda_{2}\right) B^{\frac{1}{q}}\left(\lambda_{1}, \lambda-\lambda_{1}\right)$ is expressible as a convergent single integral $B\left(\frac{\lambda-\lambda_{2}}{p}+\frac{\lambda_{1}}{q}, \frac{\lambda-\lambda_{1}}{q}+\frac{\lambda_{2}}{p}\right)$, then (17) retains the form of equality. In view of the proof of Lemma 5 , it follows that $\lambda_{1}+\lambda_{2}=\lambda$.

(iv) $\Rightarrow$ (i). If $\lambda_{1}+\lambda_{2}=\lambda$, then the following is the case:

$$
B^{\frac{1}{p}}\left(\lambda_{2}, \lambda-\lambda_{2}\right) B^{\frac{1}{q}}\left(\lambda_{1}, \lambda-\lambda_{1}\right)=B\left(\lambda_{1}, \lambda_{2}\right),
$$

which is independent of $p, q$. Hence, it follows that (i) $\Leftrightarrow(\mathrm{ii}) \Leftrightarrow$ (iv).

(iii) $\Rightarrow$ (iv). By Lemma 5, we have $\lambda_{1}+\lambda_{2}=\lambda$.

(iv) $\Rightarrow$ (iii). By Lemma 4 , if $\lambda_{1}+\lambda_{2}=\lambda$, then the following is the case:

$$
B^{\frac{1}{p}}\left(\lambda_{2}, \lambda-\lambda_{2}\right) B^{\frac{1}{q}}\left(\lambda_{1}, \lambda-\lambda_{1}\right)\left(=B\left(\lambda_{1}, \lambda_{2}\right)\right)
$$

which is the best possible constant factor in (14). Therefore, we have (iii) $\Leftrightarrow$ (iv).

Hence, we conclude that the statements (i), (ii), (iii) and (iv) are equivalent. The proof for Theorem 2 is complete.

Remark 3. In the following, we illustrate how the inequality obtained can generate some new Hardy-Hilbert-type inequalities.

(i) Taking $\eta_{1}=\eta_{2}=0$, inequality (20) reduces to the equivalent form of inequality (7) below.

$$
\left\{\sum_{n=1}^{\infty} n^{p \lambda_{2}-1}\left[\sum_{m=1}^{\infty} \frac{1}{(m+n)^{\lambda}} a_{m}\right]^{p}\right\}^{\frac{1}{p}}<B\left(\lambda_{1}, \lambda_{2}\right)\left[\sum_{m=1}^{\infty} m^{p\left(1-\lambda_{1}\right)-1} a_{m}^{p}\right]^{\frac{1}{p}} .
$$

Hence, (18) (resp. (20)) is an extension of (21).

(ii) Taking $\eta_{1}=\eta_{2}=0, \lambda_{1}=\lambda_{2}=\frac{\lambda}{2} \in\left(0, \frac{3}{2}\right](0<\lambda \leq 3)$ in inequalities (14) and (20), respectively, we have the following equivalent inequalities with the best possible constant factor $B\left(\frac{\lambda}{2}, \frac{\lambda}{2}\right)$.

$$
\begin{gathered}
\sum_{n=1}^{\infty} \sum_{m=1}^{\infty} \frac{a_{m} b_{n}}{(m+n)^{\lambda}}<B\left(\frac{\lambda}{2}, \frac{\lambda}{2}\right)\left[\sum_{m=1}^{\infty} m^{p\left(1-\frac{\lambda}{2}\right)-1} a_{m}^{p}\right]^{\frac{1}{p}}\left[\sum_{n=1}^{\infty} n^{q\left(1-\frac{\lambda}{2}\right)-1} b_{n}^{q}\right]^{\frac{1}{q}}, \\
\left\{\sum_{n=1}^{\infty} n^{\frac{p \lambda}{2}-1}\left[\sum_{m=1}^{\infty} \frac{1}{(m+n)^{\lambda}} a_{m}\right]^{p}\right\}^{\frac{1}{p}}<B\left(\frac{\lambda}{2}, \frac{\lambda}{2}\right)\left[\sum_{m=1}^{\infty} m^{p\left(1-\frac{\lambda}{2}\right)-1} a_{m}^{p}\right]^{\frac{1}{p}} .
\end{gathered}
$$

(iii) Taking $\eta_{1}=\eta_{2}=\frac{1}{4}, \lambda_{1}=\lambda_{2}=\frac{\lambda}{2} \in\left(0, \frac{3}{2}\right](0<\lambda \leq 3)$ in inequalities (14) and (20), respectively, we have the following equivalent inequalities with the best possible constant factor $B\left(\frac{\lambda}{2}, \frac{\lambda}{2}\right)$.

$$
\begin{gathered}
\sum_{n=1}^{\infty} \sum_{m=1}^{\infty} \frac{a_{m} b_{n}}{\left(m+n-\frac{1}{2}\right)^{\lambda}}<B\left(\frac{\lambda}{2}, \frac{\lambda}{2}\right)\left[\sum_{m=1}^{\infty}\left(m-\frac{1}{4}\right)^{p\left(1-\frac{\lambda}{2}\right)-1} a_{m}^{p}\right]^{\frac{1}{p}}\left[\sum_{n=1}^{\infty}\left(n-\frac{1}{4}\right)^{q\left(1-\frac{\lambda}{2}\right)-1} b_{n}^{q}\right]^{\frac{1}{q}}, \\
\left\{\sum_{n=1}^{\infty}\left(n-\frac{1}{4}\right)^{\frac{p \lambda}{2}-1}\left[\sum_{m=1}^{\infty} \frac{1}{\left(m+n-\frac{1}{2}\right)^{\lambda}} a_{m}\right]^{p}\right\}^{\frac{1}{p}}<B\left(\frac{\lambda}{2}, \frac{\lambda}{2}\right)\left[\sum_{m=1}^{\infty}\left(m-\frac{1}{4}\right)^{p\left(1-\frac{\lambda}{2}\right)-1} a_{m}^{p}\right]^{\frac{1}{p}} .
\end{gathered}
$$

In particular, by choosing $\lambda=3$ in inequalities (24) and (25), respectively, we have the following inequalities.

$$
\sum_{n=1}^{\infty} \sum_{m=1}^{\infty} \frac{a_{m} b_{n}}{\left(m+n-\frac{1}{2}\right)^{3}}<\frac{\pi}{8}\left[\sum_{m=1}^{\infty}\left(m-\frac{1}{4}\right)^{-\frac{p}{2}-1} a_{m}^{p}\right]^{\frac{1}{p}}\left[\sum_{n=1}^{\infty}\left(n-\frac{1}{4}\right)^{-\frac{q}{2}-1} b_{n}^{q}\right]^{\frac{1}{q}},
$$




$$
\left\{\sum_{n=1}^{\infty}\left(n-\frac{1}{4}\right)^{\frac{3 p}{2}-1}\left[\sum_{m=1}^{\infty} \frac{1}{\left(m+n-\frac{1}{2}\right)^{3}} a_{m}\right]^{p}\right\}^{\frac{1}{p}}<\frac{\pi}{8}\left[\sum_{m=1}^{\infty}\left(m-\frac{1}{4}\right)^{-\frac{p}{2}-1} a_{m}^{p}\right]^{\frac{1}{p}} .
$$

\section{Operator Expressions}

We set functions $\phi(m):=\left(m-\eta_{1}\right)^{p\left[1-\left(\frac{\lambda-\lambda_{2}}{p}+\frac{\lambda_{1}}{q}\right)\right]-1}, \psi(n):=\left(n-\eta_{2}\right)^{q\left[1-\left(\frac{\lambda-\lambda_{1}}{q}+\frac{\lambda_{2}}{p}\right)\right]-1}$, then $\psi^{1-p}(n)=\left(n-\eta_{2}\right)^{p\left(\frac{\lambda-\lambda_{1}}{q}+\frac{\lambda_{2}}{p}\right)-1}(m, n \in \mathrm{N})$.

Definition 1. Define the following real normed spaces.

$$
\begin{aligned}
& l_{p, \phi}:=\left\{a=\left\{a_{m}\right\}_{m=1}^{\infty} ;\|a\|_{p, \phi}:=\left(\sum_{m=1}^{\infty} \phi(m)\left|a_{m}\right|^{p}\right)^{\frac{1}{p}}<\infty\right\}, \\
& l_{q, \psi}:=\left\{b=\left\{b_{n}\right\}_{n=1}^{\infty} ;\|b\|_{q, \psi}:=\left(\sum_{n=1}^{\infty} \psi(n)\left|b_{n}\right|^{q}\right)^{\frac{1}{q}}<\infty\right\}, \\
& l_{p, \psi^{1-p}}:=\left\{c=\left\{c_{n}\right\}_{n=1}^{\infty} ;\|c\|_{p, \psi^{1-p}}:=\left(\sum_{n=1}^{\infty} \psi^{1-p}(n)\left|c_{n}\right|^{p}\right)^{\frac{1}{p}}<\infty\right\} .
\end{aligned}
$$

By assuming that $a \in l_{p, \phi}$ and setting the following case:

$$
c=\left\{c_{n}\right\}_{n=1}^{\infty}, c_{n}:=\sum_{m=1}^{\infty} \frac{1}{(m+n-\eta)^{\lambda}} a_{m}, n \in \mathrm{N},
$$

we can rewrite inequality (18) as follows:

$$
\|c\|_{p, \psi^{1-p}}<\left(B\left(\lambda_{2}, \lambda-\lambda_{2}\right)\right)^{\frac{1}{p}}\left(B\left(\lambda_{1}, \lambda-\lambda_{1}\right)\right)^{\frac{1}{q}}\|a\|_{p, \phi}<\infty,
$$

namely, $c \in l_{p, \psi^{1-p}}$.

Definition 2. Define a more accurate Hardy-Hilbert's operator $T: l_{p, \phi} \rightarrow l_{p, \psi^{1-p}}$ as follows: For any $a \in l_{p, \phi}$, there exists a unique representation $c \in l_{p, \psi^{1-p}}$. Define the formal inner product of Ta and $b \in l_{q, \psi}$ and the norm of $T$ as follows.

$$
(T a, b):=\sum_{n=1}^{\infty}\left[\sum_{m=1}^{\infty} \frac{1}{(m+n-\eta)^{\lambda}} a_{m}\right] b_{n},\|T\|:=\sup _{a(\neq 0) \in l_{p, \phi}} \frac{\|T a\|_{p, \psi^{1-p}}}{\|a\|_{p, \phi}} .
$$

As a direct consequence of Theorems 1 and 2, we obtain the following case.

Theorem 3. If $a \in l_{p, \phi}, b \in l_{q, \psi},\|a\|_{p, \phi},\|b\|_{q, \psi}>0$, then we have the following equivalent inequalities.

$$
\begin{aligned}
& (T a, b)<\left(B\left(\lambda_{2}, \lambda-\lambda_{2}\right)\right)^{\frac{1}{p}}\left(B\left(\lambda_{1}, \lambda-\lambda_{1}\right)\right)^{\frac{1}{q}}\|a\| p, \phi\|b\|_{q, \psi}, \\
& \|T a\|_{p, \psi^{1-p}}<\left(B\left(\lambda_{2}, \lambda-\lambda_{2}\right)\right)^{\frac{1}{p}}\left(B\left(\lambda_{1}, \lambda-\lambda_{1}\right)\right)^{\frac{1}{q}}\|a\|_{p, \phi} .
\end{aligned}
$$

Moreover, if $\lambda_{1}+\lambda_{2}=\lambda$, then the constant factor $\left(B\left(\lambda_{2}, \lambda-\lambda_{2}\right)\right)^{\frac{1}{p}}\left(B\left(\lambda_{1}, \lambda-\lambda_{1}\right)\right)^{\frac{1}{q}}$ in (28) and (29) is the best possible; namely, the following is the case. 


$$
\|T\|=B\left(\lambda_{1}, \lambda_{2}\right)
$$

If the constant factor $\left(B\left(\lambda_{2}, \lambda-\lambda_{2}\right)\right)^{\frac{1}{p}}\left(B\left(\lambda_{1}, \lambda-\lambda_{1}\right)\right)^{\frac{1}{q}}$ in (28) (or (29)) is the best possible, then for $\lambda-\lambda_{1}-\lambda_{2} \in\left[\left(\lambda-\lambda_{1}-\frac{3}{2}\right) p,\left(\frac{3}{2}-\lambda_{1}\right) p\right]$, we have $\lambda-\lambda_{1}-\lambda_{2}=0$, namely, $\lambda_{1}+\lambda_{2}=\lambda$.

\section{Conclusions}

In this paper, by means of the construction of weight coefficients, the idea of introduced parameters, the techniques of real analysis and with the help of the Euler-Maclaurin summation formula, a more accurate extension of Hardy-Hilbert's inequality is established in Theorem 1, which contains parameters composed of a pair of weight coefficients with their sum. The equivalent conditions of the best possible constant factor related to several parameters are provided in Theorem 2. We also consider the equivalent forms, the operator expressions and some particular inequalities in Theorem 3 and Remark 3. The results provided in lemmas and theorems provide a significant supplement to the inequalities of the Hardy-Hilbert type. For further study, we may use these results and methods to establish new Hardy-Hilbert's inequalities involving partial sums, and this would enable us to confront the extensions of the results obtained in [21]. We will also investigate some new generalizations of Hardy-Hilbert's inequality by using the extended Euler-Maclaurin summation formula by using the techniques of analytical inequalities (cf. [22,23]).

Author Contributions: B.Y. carried out mathematical studies and participated in drafting the manuscript. S.W. and X.H. participated in the design of the study and performed numerical analysis. All authors contributed equally in the preparation of this paper. All authors have read and agreed to the published version of the manuscript.

Funding: This work is supported by the National Natural Science Foundation (No.11961021), the Hechi University Research Foundation for Advanced Talents under Grant (2021GCC024) and the Guangxi Natural Science Foundation (No. 2020GXNSFAA159084).

Institutional Review Board Statement: Not applicable.

Informed Consent Statement: Not applicable.

Data Availability Statement: The data used to support the findings of this study are included within the article.

Conflicts of Interest: The authors declare no conflict of interest.

\section{References}

1. Hardy, G.H.; Littlewood, J.E.; Polya, G. Inequalities; Cambridge University Press: Cambridge, UK, 1934.

2. Yang, B.C. On Hilbert's integral inequality. J. Math. Anal. Appl. 1998, 220, 778-785.

3. Yang, B.C. A note on Hilbert's integral inequality. Chin. Quart. J. Math. 1998, 13, 83-86.

4. Yang, B.C. On a generalization of Hilbert double series theorem. J. Nanjing Univ. Math. Biquarterly 2001, 18, 145-152.

5. Yang, B.C.; Debnath, L. On the extended Hardy-Hilbert's inequality. J. Math. Anal. Appl. 2002, 272, 187-199. [CrossRef]

6. Krnić, M.; Pečarić, J. Extension of Hilbert's inequality. J. Math. Anal. Appl. 2006, 324, 150-160. [CrossRef]

7. Huang, X.Y.; Wu, S.H.; Yang, B.C. A more accurate half-discrete Hilbert-type inequality involving one upper limit function and one partial sum. Symmetry 2021, 13, 1548. [CrossRef]

8. Yang, B.C. The Norm of Operator and Hilbert-Type Inequalities; Science Press: Beijing, China, 2009.

9. Krnić, M.; Pečarić, J. General Hilbert's and Hardy's inequalities. Math. Inequal. Appl. 2005, 8, 29-51. [CrossRef]

10. Perić, I.; Vuković, P. Multiple Hilbert's type inequalities with a homogeneous kernel. Banach J. Math. Anal. $2011,5,33-43$. [CrossRef]

11. Huang, Q.L. A new extension of Hardy-Hilbert-type inequality. J. Inequal. Appl. 2015, 2015, 397. [CrossRef]

12. He, B. A multiple Hilbert-type discrete inequality with a new kernel and best possible constant factor. J. Math. Anal. Appl. 2015, 431, 889-902. [CrossRef]

13. Xu, J.S. Hardy-Hilbert's inequalities with two parameters. Adv. Math. 2007, 36, 63-76.

14. Xie, Z.T.; Zeng, Z.; Sun, Y.F. A new Hilbert-type inequality with the homogeneous kernel of degree -2. Adv. Appl. Math. Sci. 2013, 12,391-401. 
15. Zheng, Z.; Gandhi, R.R.; Xie, Z.T. A new Hilbert-type inequality with the homogeneous kernel of degree -2 and with the integral. Bull. Math. Sci. Appl. 2014, 3, 11-20. [CrossRef]

16. Xin, D.M. A Hilbert-type integral inequality with the homogeneous kernel of zero degree. Math. Theory Appl. 2010, 30, 70-74.

17. Azar, L.E. The connection between Hilbert and Hardy inequalities. J. Inequal. Appl. 2013, 2013, 452. [CrossRef]

18. Adiyasuren, V.; Batbold, T.; Krnić, M. Hilbert-type inequalities involving differential operators, the best constants, and applications. Math. Inequal. Appl. 2015, 18, 111-124. [CrossRef]

19. Hong, Y.; Wen, Y.M. A necessary and sufficient condition of that Hilbert type series inequality with homogeneous kernel has the best constant factor. Ann. Math. 2016, 37, 329-336.

20. Kuang, J.C. Applied Inequalities; Shangdong Science and Technology Press: Jinan, China, 2004.

21. Adiyasuren, V.; Batbold, T.; Azar, L.E. A new discrete Hilbert-type inequality involving partial sums. J. Inequal. Appl. 2019, 2019, 127. [CrossRef]

22. Agarwal, P.; Dragomir, S.S.; Jleli, M.; Samet, B. Advances in Mathematical Inequalities and Applications; Birkhäuser: Basel, Switzerland, 2018

23. Masjed-Jamei, M.; Beyki, M.R.; Koepf, W. An extension of the Euler-Maclaurin quadrature formula using a parametric type of Bernoulli polynomials. Bull. Sci. Math. 2019, 156, 102798. [CrossRef] 